\title{
COMPORTAMENTO INICIAL DE ESPÉCIES NA REVEGETAÇÃO DA MATA DE GALERIA NA FAZENDA MANDAGUARI, EM INDIANÓPOLIS, MG ${ }^{1}$
}

\begin{abstract}
Júlia Araújo de Lima², Denise Garcia de Santana³ e Mauro Eloi Nappo ${ }^{4}$
RESUMO - As áreas degradadas demandam prioridade nas ações de revegetação, e os estudos ainda são escassos ou insuficientes para orientar, de forma efetiva, as práticas de recuperação. Os objetivos foram verificar o crescimento, desenvolvimento e capacidade de sobrevivência de indivíduos das espécies implantadas na área de preservação permanente no entorno do reservatório da Fazenda Mandaguari, em Indianópolis, Minas Gerais, como subsídio para programas de recuperação de ambientes em condições similares. Espécies observadas em fragmentos de vegetação natural nas áreas da fazenda foram a base para a seleção e produção das mudas utilizadas na revegetação. A revegetação foi realizada entre 2005 e 2006 em uma área de 2,34 ha, na qual foram alocadas 26 parcelas, medindo $21 \times 21 \mathrm{~m}\left(441 \mathrm{~m}^{2}\right)$. A distribuição das parcelas e dos indivíduos das espécies por parcela foi aleatória, com algumas restrições na casualização para alocar espécies indiferentes ou, mesmo, resistentes ao alagamento próximas à margem da represa. As parcelas diferenciaram-se entre si pela composição percentual dos grupos ecológicos (fase sucessional) e pelo espaçamento ( 3 × 3 m e 3 x 6 m). Guazuma ulmifolia e Aegiphila lhotzkiana, apesar de suas taxas de mortalidade relativamente altas, 50 e 28,6\%, respectivamente, agregaram características importantes de desenvolvimento que são a capacidade de cobertura (incrementos anuais em altura de 160 e $155 \mathrm{~cm}$, respectivamente) e estabelecimento (incrementos anuais em diâmetro de 40,6 e 36,3 mm, respectivamente). Portanto, foram qualificadas como edificantes do processo de regeneração. Chorisia speciosa, apesar do pouco destaque no incremento em altura $(71 \mathrm{~cm})$, investiu mais no crescimento diamétrico $(31,4 \mathrm{~mm})$, característica fundamental para o estabelecimento da espécie.
\end{abstract}

Palavras-chave: Mata Ciliar, recuperação de área degradada e sucessão.

\section{INITIAL BEHAVIOR OF SPECIES IN REVEGETATION OF THE GALLERY FOREST IN THE MANDAGUARI FARM IN INDIANÓPOLIS, MG}

\begin{abstract}
Degraded areas demand priority regarding actions for revegetation and recovery. However, there is a scarcity of studies to effectively guide us in these recovery practices. The objective of this work was to verify the growth, development and survival capacity of the individuals of the species implanted in the area of permanent preservation around the water reservoir on Mandaguari Farm, in Indianópolis, Minas Gerais. The species observed in fragments of the natural vegetation in the areas of the farm were the basis for the selection and production of seedlings used in the replanting. The replanting was carried out between 2005 and 2006 in $2.34 \mathrm{ha}$, which was divided in 26 parcels, measuring $21 \times 21 \mathrm{~m}\left(441 \mathrm{~m}^{2}\right)$. The distribution of the species was randomly performed with some restrictions in the randomization, so as to allocate neutral or even resistant species to flooding close to the margin of the dam. The areas differed from each other as to the percentile composition of the ecological groups (succession) and spacing ( $3 \times 3$ and $3 \times 6 \mathrm{~m}$ ). In spite of their relatively high mortality rates, Guazuma ulmifolia and Aegiphila lhotzkiana (50 and 28.6\%, respectively) aggregated important development characteristics, which are their covering capacity (increments in height of 160 and $155 \mathrm{~cm}$, respectively) and establishment (increments in diameter of 40.6 and $36.3 \mathrm{~mm}$, respectively). Therefore, they were qualified as edifying agents in the regeneration process. Chorisia speciosa, in spite of the little prominence in height increment $(71 \mathrm{~cm})$, invested more in the diametric growth $(31.4 \mathrm{~mm})$, which is a fundamental characteristic for the establishment of the species.
\end{abstract}

Keywords: Riparian forest, recovery of degraded area and succession.

\footnotetext{
${ }^{1}$ Recebido em 03.10.2007 e aceito para publicação em 29.05.2009.

${ }^{2}$ Programa de Pós-Graduação em Agronomia da UFU. E-mail: <juliaaraujo@yahoo.com.br>.

${ }^{3}$ Instituto de Ciências Agrárias da Universidade Federal de Uberlândia (UFU). E-mail: <dgsantana@ umuarama.ufu.br>.

${ }^{3}$ Departamento de Engenharia Florestal da Universidade de Brasília (UNB). E-mail: <mauronappo@yahoo.com.br>.
} 


\section{INTRODUÇÃO}

Apesar da crescente consciência sobre a importância da recuperação dos recursos florestais e da necessidade de conservá-los, em especial os que têm funções específicas e que ocupam áreas de preservação permanente, como as Matas Ciliar e de Galeria, os estudos que orientam e avaliam a revegetação dessas áreas ainda são escassos ou insuficientes em face da complexidade dos processos ecológicos envolvidos. No Estado de Minas Gerais, os estudos de vegetação estão concentrados na composição florística e na estrutura fitossociológica de remanescentes bem preservados, podendo citar os levantamentos de Oliveira Filho e Martins (1986), Oliveira Filho (1989), Oliveira Filho et al. (1994), Araújo et al. (1997) e Pedralli e Teixeira (1997). Em relação a estudos de estruturação e avaliação de povoamentos mistos com espécies autóctones para a reabilitação ou restauração de áreas degradadas existe grande lacuna de conhecimento que permita orientar a seleção de espécies e as práticas silviculturais a serem empregadas. Comumente é utilizado reduzido número de espécies para ambientes distintos, fato que dificulta e coloca em risco a função da revegetação, por promoverem baixa diversidade de espécies, genética e de ambientes, conforme observado por White e Pickett (1985) e Kageyama (1987).

Especialmente para Matas de Galeria, a recuperação requer a utilização de princípios ecológicos e silviculturais oriundos do conhecimento científico existente para melhor nortear a definição de modelos de recuperação. Soma-se a isso o fato de as Matas de Galeria se encontrarem em constante transição quanto aos atributos físicos e químicos do solo e os gradientes de umidade em relação ao meio circunvizinho (REZENDE, 1998). Por meio dessas inter-relações são formados padrões estruturais, espaciais e temporais nas comunidades biológicas (PINTO-COELHO, 2000), sendo estes os modelos que permitem, no médio e longo prazos, a sustentabilidade de programas de recuperação de áreas degradadas, em especial para a restauração de ambientes que são áreas de preservação permanente.

A atividade de revegetação fornece os ingredientes iniciais necessários para o início de um processo de recuperação. A manutenção e a proteção dessas áreas darão condições para que o meio se encarregue da continuidade dos processos ecológicos mais detalhados (MACEDO et al., 1993), a natureza fica com os ajustes mais finos (KAGEYAMA e GANDARA, 2000). Para

R. Árvore, Viçosa-MG, v.33, n.4, p.685-694, 2009 que a revegetação em uma área ocorra com sucesso, Fonseca et al. (2001) enfatizaram que a incorporação de vegetação heterogênea com espécies autóctones da fitofisionomia da área, favorece a continuidade das funções específicas destas na comunidade. Nesse aspecto, visando gerar conhecimento prévio sobre as espécies presentes na vegetação pré-existente, é indicado o levantamento florístico em fragmentos de matas remanescentes nas bacias hidrográficas que compõem as Matas Ciliares (FONSECA et al., 2001; FERREIRA e DIAS, 2004). Num programa de recuperação de ambientes degradados, vários tipos de revegetação podem ser planejados, dependendo, basicamente, das potencialidades locais e dos objetivos a serem atingidos (ANDRADE, 1991).

Em propriedades rurais, as Matas Ciliares e de Galeria são utilizadas como pontos para a construção de reservatórios de água para irrigação ou para outras finalidades e, como tal, são consideradas áreas de preservação permanente, conforme considerado originalmente no Código Florestal Brasileiro (Lei 4.771, de 1965) e demais disciplinamentos legais. Como a edificação dessas obras (barramentos) provoca o aumento do tamanho do corpo d'água e, em muitos casos, a abertura de áreas para empréstimo, é necessário proceder à recomposição da área para proteção dos recursos hídricos, para continuidade dos corredores de dispersão da flora e da fauna que colonizam esses ambientes. Assim, pesquisas têm sido realizadas visando adequar métodos e técnicas de revegetação às diferentes situações, porém ainda é necessário o seu aprimoramento de tal sorte que todos os investimentos realizados sejam efetivamente capazes de se sustentarem. Esses processos devem promover as mais estreitas e complexas relações entre solo-planta-animal (GISLER e BARBOSA, 2000).

Diante do exposto, os objetivos do trabalho foram verificar o crescimento, desenvolvimento e capacidade de sobrevivência de indivíduos das espécies implantadas na área de preservação permanente no entorno do reservatório de água da Fazenda Mandaguari, em Indianópolis, Minas Gerais, originalmente abrigada por uma Mata de Galeria, como subsídio para programas de recuperação de ambientes em condições similares.

\section{MATERIAL E MÉTODOS}

\subsection{Caracterização da área}

A revegetação da área de Mata de Galeria no reservatório de água para irrigação, da Fazenda 
Mandaguari, foi realizada entre 2005 e 2006 em uma área de 2,34 ha localizada no Município de Indianópolis, Minas Gerais (1900'04" S e 48 20' 17' W). A área de estudo é composta pela margem esquerda do reservatório formado pela extensão de uma nascente. O clima da região é, segundo a classificação climática de Köppen (1948), do tipo $\mathrm{Cw}_{\mathrm{a}}$ - tropical de altitude, com inverno seco entre os meses de abril a setembro e verão chuvoso entre novembro e março (RIBEIRO e WALTER, 1998). A temperatura média anual é de $26^{\circ} \mathrm{C}$ e a precipitação pluviométrica em torno de 1.200 $\mathrm{mm}$ por ano; e umidade relativa do ar entre 50 e $60 \%$ na estação seca e 85 e $90 \%$ no inverno. No mês da implantação (dezembro de 2005), a precipitação média foi de $539 \mathrm{~mm}$.

A revegetação foi realizada em uma faixa de $50 \mathrm{~m}$ da margem esquerda do reservatório, com gradiente de altitude variando de 940 a 948 m. O solo da área foi classificado como Latossolo Vermelho textura argilosa, com aproximadamente $60 \%$ de argila (EMBRAPA e EPAMIG, 1982). Amostras do solo (camada de 0-20 cm) foram coletadas antes da implantação e levadas para o Laboratório de Análises de Solo e Fertilizantes da Universidade Federal de Uberlândia.

\subsection{Seleção das espécies, germinação de sementes e produção de mudas}

Espécies com ocorrência natural em fragmentos de vegetação nas áreas da fazenda foram à base para a seleção e produção das mudas utilizadas na revegetação. Como critério de seleção das espécies, consideraram-se a produtividade, disponibilidade de sementes, crescimento e desenvolvimento e adequação às características da área de implantação. Outras espécies com sementes não coletadas na área, porém aptas para o local de revegetação, foram também incorporadas (Tabela 1).

Tabela 1 - Espécies cultivadas em viveiro e implantadas na área revegetada da mata de galeria da Fazenda Mandaguari no Município de Indianópolis, MG. Os grupos ecológicos P: pioneira, P(si): pioneira secundaria inicial, NP: não pioneira; CE: espécies de cerrado; as indicações são A: áreas encharcadas permanentes, B: áreas com inundação temporária e C: áreas bem drenadas, não alagadas.

Table 1 - Species cultivated in nursery and implanted in the area revegetada of the forest of gallery of Fazenda Mandaguari, in the Municipal district of Indianópolis, MG. The ecological groups $P$ : pioneer, $P($ si): pioneer would support initial, NP: no pioneer; AND CE: species of Savannah. The indications are THE: permanent areas, B: areas with temporary flood and $C$ : areas well drained, no flooded

\begin{tabular}{|c|c|c|c|}
\hline Família & Espécie & Grupo ecológico & Indicação \\
\hline Anacardiaceae & Anacardium humile St. Hil. & $\mathrm{CE}$ & $\mathrm{C}$ \\
\hline Anacardiaceae & Anacardium occidentale L. & $\mathrm{P}$ & $\mathrm{C}$ \\
\hline Anacardiaceae & Astronium fraxinifolium Schott. & $\mathrm{P}$ & $\mathrm{C}$ \\
\hline Anacardiaceae & Lithraea molleoides Engl. & $\mathrm{P}(\mathrm{si})$ & B \\
\hline Anacardiaceae & Tapirira guianensis Aubl. & $\mathrm{P}(\mathrm{si})$ & $\mathrm{A}, \mathrm{B}$ \\
\hline Apocynaceae & Aspidosperma cylindrocarpum Müll. Arg. & NP & $\mathrm{B}, \mathrm{C}$ \\
\hline Apocynaceae & Aspidosperma macrocarpon Mart. & $\mathrm{CE}$ & $\mathrm{C}$ \\
\hline Apocynaceae & Aspidosperma parvifolium A. DC. & NP & $\mathrm{C}$ \\
\hline Apocynaceae & Aspidosperma tomentosum Mart. & NP & $\mathrm{C}$ \\
\hline Bignoniaceae & Jacaranda cuspidifolia Mart. & $\mathrm{P}$ & $\mathrm{C}$ \\
\hline Bombacaceae & Chorisia speciosa A. St.- Hil. & $\mathrm{P}(\mathrm{si})$ & $\mathrm{B}, \mathrm{C}$ \\
\hline Bombacaceae & Eriotheca gracilipes K. Schum. & $\mathrm{CE}$ & $\mathrm{C}$ \\
\hline Clusiaceae & Kielmeyera coriacea Mart. & $\mathrm{CE}$ & $\mathrm{B}, \mathrm{C}$ \\
\hline Ebenaceae & Diospyros burchellii Hiern & $\mathrm{CE}$ & $\mathrm{B}, \mathrm{C}$ \\
\hline Fabaceae & Anadenanthera colubrina (Vell) Brenan & $\mathrm{P}$ & $\mathrm{B}, \mathrm{C}$ \\
\hline Fabaceae & Dalbergia miscolobium Benth. & $\mathrm{CE}$ & $\mathrm{C}$ \\
\hline Fabaceae & Dipteryx alata Vogel & NP & $\mathrm{C}$ \\
\hline Fabaceae & Machaerium opacum Vog. & NP & $\mathrm{C}$ \\
\hline Fabaceae & Ormosia arborea Harms & NP & $\mathrm{C}$ \\
\hline Leguminosae Fabaceae & Platypodium elegans Vog. & $\mathrm{P}$ & $\mathrm{B}, \mathrm{C}$ \\
\hline Leguminosae Fabaceae & Senna multijuga ( L.C.Richard) & $\mathrm{P}(\mathrm{si})$ & $\mathrm{C}$ \\
\hline Leguminosae & Piptadenia gonoacantha Macbride & NP & $\mathrm{C}$ \\
\hline Leguminosae & Acosmium dasycarpum (Vog.) Yakovl. & $\mathrm{CE}$ & $\mathrm{C}$ \\
\hline
\end{tabular}


Tabela 1 - Cont.

Table 1 - Cont.

\begin{tabular}{|c|c|c|c|}
\hline Família & Espécie & Grupo ecológico & Indicação \\
\hline Leguminosae & Inga sessilis Mart. & NP & $\mathrm{B}, \mathrm{C}$ \\
\hline Lecythidaceae & Cariniana estrellensis Kuntze & NP & $\mathrm{C}$ \\
\hline Leguminosae & Acacia polyphylla Clos & $\mathrm{P}$ & $\mathrm{C}$ \\
\hline Leguminosae & Anadenanthera peregrina Speg. & $\mathrm{P}$ & B \\
\hline Leguminosae & Dimorphandra mollis Benth. & $\mathrm{CE}$ & $\mathrm{C}$ \\
\hline Leguminosae & Stryphnodendron adstringens (Mart.) Coville & $\mathrm{CE}$ & $\mathrm{C}$ \\
\hline Leguminosae-Caesalpinoideae & Hymenaea courbaril L. & NP & $\mathrm{B}, \mathrm{C}$ \\
\hline Leguminosae-Caesalpinoideae & Enterolobium contortisiliquum (Vell.) Morong & $\mathrm{P}(\mathrm{si})$ & $\mathrm{B}, \mathrm{C}$ \\
\hline Leguminosae-Caesalpinoideae & Bauhinia longifolia D. Dietr. & NP & $\mathrm{C}$ \\
\hline Leguminosae-Caesalpinoideae & Copaifera langsdorffii Desf. & NP & $\mathrm{C}$ \\
\hline Leguminosae-Mimosoideae & Inga laurina willd. & NP & $\mathrm{A}, \mathrm{B}$ \\
\hline Leguminosae-Mimosoideae & Enterolobium gummiferum Macbride & $\mathrm{CE}$ & $\mathrm{C}$ \\
\hline Melastomataceae & Miconia ferruginata DC. & $\mathrm{P}$ & $\mathrm{C}$ \\
\hline Meliaceae & Cedrela fissilis Vell. & $\mathrm{P}$ & $\mathrm{C}$ \\
\hline Myristicaceae & Virola sebifera Aubl. & NP & $\mathrm{B}, \mathrm{C}$ \\
\hline Myrsinaceae & Myrsine umbellata Mart. & $\mathrm{P}(\mathrm{si})$ & $\mathrm{A}, \mathrm{B}, \mathrm{C}$ \\
\hline Rubiaceae & Genipa americana $\mathrm{L}$. & NP & A,B \\
\hline Rubiaceae & Alibertia sessilis K. Schum. & NP & $\mathrm{B}, \mathrm{C}$ \\
\hline Rutaceae & Zanthoxylum rhoifolium Lam. & $\mathrm{P}(\mathrm{si})$ & $\mathrm{C}$ \\
\hline Sapotaceae & Pouteria torta Radlk. & NP & A \\
\hline Sterculiaceae & Guazuma ulmifolia Lam. & $\mathrm{P}$ & $\mathrm{C}$ \\
\hline Ulmaceae & Trema micrantha (L.) Blume & $\mathrm{P}(\mathrm{si})$ & $\mathrm{C}$ \\
\hline Verbenaceae & Aegiphila lhotskiana Cham. & $\mathrm{CE}$ & $\mathrm{C}$ \\
\hline Verbenaceae & Aegiphila sellowiana Cham. & $\mathrm{P}$ & $\mathrm{C}$ \\
\hline
\end{tabular}

As sementes dormentes foram germinadas em câmara de germinação e as demais, semeadas diretamente em bandejas multicelulares com vermiculita e substrato comercial (Plantmax $®$ ) na proporção 1:3 e dispostas no viveiro até a fase de plântula. As sementes germinadas e as plântulas foram repicadas para sacos polietileno (1 L) contendo solo e areia peneirados, na proporção de $3: 1$ (v/v), fumigados por $48 \mathrm{~h}$. As mudas foram adubadas três meses antes do plantio e levadas para aclimatação até o final de novembro, época da implantação.

\subsection{Implantação e manejo}

$\mathrm{Na}$ área foram marcadas 26 parcelas medindo 21 x $21 \mathrm{~m}\left(441 \mathrm{~m}^{2}\right)$, distribuídas paralelamente à margem da represa, obedecendo-se a distância mínima de $3 \mathrm{~m}$ da borda, além da marcação de áreas de carreadores para o manejo. Dessas, 13 foram alocadas na parte norte e 13 na sul, separadas por um carreador de $5 \mathrm{~m}$ de largura. No preparo do solo, as gramíneas e as plantas infestantes foram roçadas mecanicamente a $30 \mathrm{~cm}$ do solo, deixandose a palhada como cobertura morta.

Em seguida, a área foi demarcada e estaqueada, e na base da muda foi feito um coroamento de $1 \mathrm{~m}$ de diâmetro. Em dezembro de 2005, a distribuição das espécies foi feita seguindo o método de amostragem aleatória dentro de cada parcela, sendo esta composta por no máximo dois indivíduos por espécie, com algumas restrições na casualização para alocar espécies indiferentes ou, mesmo, resistentes ao alagamento próximas à margem da represa. Cada espécie foi representada por no mínimo 10 indivíduos na área. As parcelas diferenciaram-se entre si em espaçamento (espaçamento de $3 \times 3 \mathrm{~m} \mathrm{e} 3 \times 6 \mathrm{~m}$ ), principalmente pela posição entre espécies pioneiras e não pioneiras e número de plantas.

As covas foram abertas manualmente, adubadas com $120 \mathrm{~g}$ de monoamônio fosfatado (10: 54: 0). Os tratos culturais foram realizados para o controle de formigas por meio de isca-formicida, bem como formicidas em pó. O controle foi feito dois meses antes do plantio com aplicações posteriores, a cada sete dias. A manutenção da área foi feita sempre que necessária, com capinas manuais nas faixas de plantio e coroamento, evitando-se a eliminação de indivíduos de espécies arbustivas ou arbóreas em regeneração. 


\subsection{Mortalidade, crescimento e desenvolvimento das plantas}

A primeira avaliação do percentual de pegamento, crescimento e desenvolvimento das mudas foi efetuada aos 36 dias após o plantio. Devido ao alto percentual de mortalidade de algumas espécies, os indivíduos avaliados foram sorteados de maneira aleatória com a marcação de, no mínimo, três indivíduos por espécie, em parcelas diferentes. As demais avaliações foram realizadas aos 4, 7, 10 e 13 meses após o plantio, sendo determinado o diâmetro na base do colo e a altura da planta. Os espaçamentos não foram analisados para os indivíduos das espécies, uma vez que o período foi insuficiente para proporcionar competição nutricional e sombreamento.

\section{RESULTADOS E DISCUSSÃO}

\subsection{Caracterização da área}

Um estudo preliminar relevou que nos dois anos antes da implantação a área estava em descanso, com predomínio de gramíneas e poucas espécies arbustivas emergindo naturalmente na sucessão. Assim, a baixa capacidade natural de autorrecuperação do ambiente se deve a esse curto período, não possibilitando o desenvolvimento das plântulas presentes no banco de sementes do solo. A proximidade da área sob revegetação com outros fragmentos próximos (área de preservação permanente e reserva legal) e dispersores presentes podem, segundo Barbosa (2002), acelerar a ligação da dinâmica natural e das constantes renovações dos ciclos de crescimento.

O período de pousio da área não foi suficiente para recuperar a composição natural do solo. Os valores da composição química do solo caracterizavam-se por vestígios de atividade agrícola recente em função dos médios valores de saturação de bases $\left(4,05 \mathrm{cmol}_{\mathrm{c}} \mathrm{dm}^{-3}\right)$, baixa acidez $(\mathrm{pH}=5,8)$ e elevados de potássio $\left(0,35 \mathrm{cmol}_{\mathrm{c}} \mathrm{dm}^{-3}\right)$, fósforo $\left(19 \mathrm{mg} \mathrm{dm}^{-3}\right)$ e cálcio $\left(3,0 \mathrm{cmol}_{\mathrm{c}} \mathrm{dm}^{-3}\right)$ na camada superficial $(0-20 \mathrm{~cm})$.

\subsection{Espécies edificantes}

O período mais crítico para o estabelecimento das mudas foi até os 30 dias após a implantação, apesar da alta pluviosidade no período $(539 \mathrm{~mm})$. A sobrevivência de plantas $(70,3 \%)$ foi considerada satisfatória (Figura 1), sendo influenciada indistintamente por espécies de mais de um grupo sucessional. A grande maioria das plantas que sobreviveram no primeiro mês permaneceu até um ano depois de implantadas, o que não implica necessariamente seu estabelecimento. Isso, segundo Holl et al. (2000), depende da probabilidade conjunta de chegar a sobreviver, vencendo barreiras como patógenos, competição, predação e herbivoria.

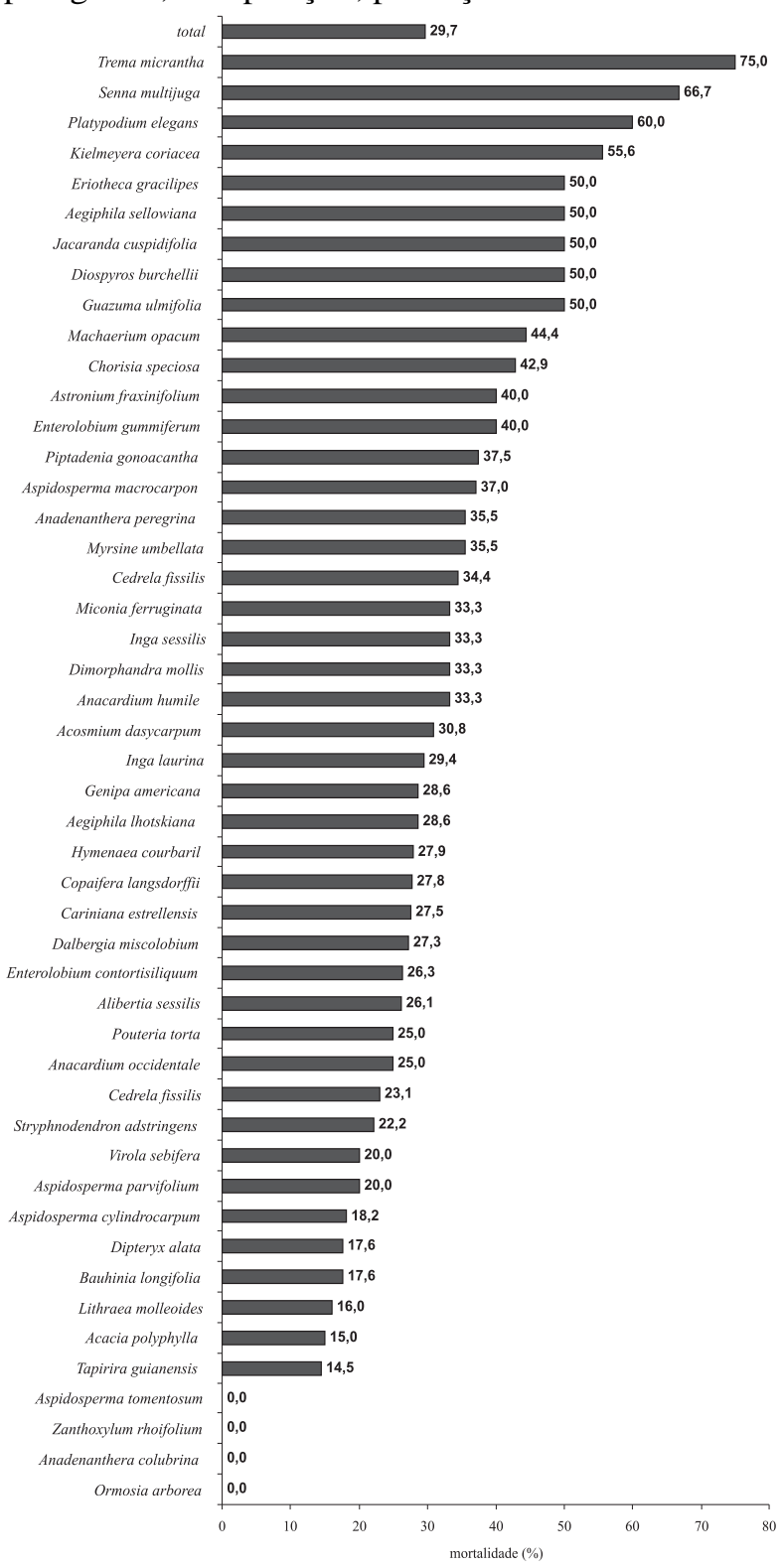

Figura 1 - Percentual de mortalidade das espécies após 36 dias da implantação na área no entorno do reservatório da Fazenda Mandaguari, no Município da Indianópolis, MG.

Figure 1 -Mortality (\%) of the species 36 days after implantation in the area around Mandaguari Farm's reservoir in the municipal district of Indianópolis, MG.

R. Árvore, Viçosa-MG, v.33, n.4, p.685-694, 2009 
A sobrevivência parece não estar diretamente relacionada ao grupo ecológico. As maiores taxas de mortalidade, entre 50 e $75 \%$, ocorreram em Trema micrantha, Senna multijuga, Platypodium elegans, Kielmeyera coriacea, Eriotheca gracilipes, Aegiphila sellowiana, Jacaranda cuspidifolia, Diospyros burchellii e Guazuma ulmifolia (Figura 1). Contudo, autores como Felfili (2000) e Martins (2001) classificaram Aegiphila sellowiana, Guazuma ulmifolia, Senna multijuga, Platypodium elegans, Jacaranda cuspidifolia e Trema micrantha como espécies pioneiras e altamente resistentes às condições de grande luminosidade, tanto para germinação quanto para o crescimento e desenvolvimento.

Possivelmente, o conjunto de características que confere às espécies pioneirismo não é a única responsável pelo estabelecimento. Apesar de as mudas terem passado por um período de três meses de aclimatação antes da implantação no campo, alguns autores questionaram sobre problemas fisiológicos. Segundo Hanba et al. (2002) e Schlüter et al. (2003), as alterações na estrutura interna foliar constituem aspectos decisivos na capacidade de aclimatação das espécies expostas a diferentes condições de ambiente. Em adição, as características fotossintéticas geralmente variam em resposta a diferentes regimes de irradiância (BOARDMAN, 1977).

A luz, principalmente no que se refere à sua intensidade, tem sido reconhecida como o fator mais importante para os mecanismos de regeneração e crescimento das florestas (AMO, 1985). A adaptação ambiental e o sucesso ou não da regeneração das espécies são importantes, principalmente, na fase juvenil por condicionar mudanças morfogenéticas e fisiológicas na sua estrutura e função (WHATLEY e WHATLEY, 1982).

As altas taxas de sobrevivência de 73,7 e 76,9\% (Figura 1) para Enterolobium contortisiliquum e Cedrela fissilis, respectivamente, podem estar relacionadas ao pioneirismo dessas espécies. Na comparação com outros trabalhos, Meneghello e Mattei (2004) encontraram para Enterolobium contortisiliquum 20,3\% de mortalidade no período de 30 dias e acumularam 12,4\% após 210 dias. Já em Cedrela fissilis houve perda de 4,9\% em 30 dias e de 12,2\% após 210 dias.

Embora a sobrevivência das espécies seja condição necessária para o sucesso da revegetação, Martins et al. (2001) salientaram que as variáveis ambientais podem interferir no comportamento das espécies. Portanto, essa variável não é suficiente para garantir que um grupo de espécies se estabeleça, se desenvolva, aumente a capacidade de cobertura do solo e inicie um processo de regeneração natural. Espécies com $100 \%$ de sobrevivência, como Ormosia arborea, Aspidosperma tomentosum, Anadenanthera colubrina e Zanthoxylum rhoifolium, formaram dois grupos distintos quanto aos incrementos em altura (Figura 2) e diâmetro (Figura 3). Anadenanthera colubrina e Zanthoxylum rhoifolium apresentaram incrementos em altura entre 58 e $93 \mathrm{~cm}$, respectivamente, ao longo de 13 meses, enquanto Ormosia arborea e Aspidosperma tomentosum foram as espécies que menos cresceram no período avaliado, com incrementos de apenas 30 e $40 \mathrm{~mm}$, respectivamente (Figura 2). Resultados similares para indivíduos dessas espécies foram observados no incremento em diâmetro (Figura 3).

No desenvolvimento das 48 espécies em campo, observou-se a formação de três grupos bem distintos, com incrementos alto (acima de $100 \mathrm{~cm}$ ), médio (entre 50 e $99 \mathrm{~cm}$ ) e baixo (menores que $50 \mathrm{~cm}$ ) em altura (Figura 2). Guazuma ulmifolia, Aegiphila lhotskiana, Anadenanthera peregrina, Enterolobium contortisiliquum, Piptadenia gonoacantha e Aegiphila sellowiana foram as espécies com maior incremento em altura durante os 13 meses, ultrapassando, em média, $100 \mathrm{~cm}$, com destaque para Guazuma ulmifolia, que atingiu $160 \mathrm{~cm}$ no período.

Quanto ao incremento em diâmetro, Guazuma ulmifolia foi a espécie com maior desenvolvimento durante os 13 meses de implantação (Figura 3), sendo seu incremento maior nos três $(14,77 \mathrm{~mm})$ e nos seis meses (11,5 mm), superando as outras espécies. No período em que as chuvas diminuíram, essa espécie continuou seu crescimento em diâmetro, destacando-se das outras. Outras espécies como Inga laurina, Lithraea molleoides, Tapirira guianensis, Enterolobium contortisiliquum, Cedrela fissilis, Aegiphila sellowiana e Chorisia speciosa tiveram incremento variando de 10 a $20 \mathrm{~mm}$ durante os nove meses. Algumas espécies morreram durante a análise de desenvolvimento, não sendo possível comparar seu desenvolvimento do primeiro ano durante as quatro estações climáticas. A maioria das espécies se desenvolveu melhor durante os três primeiros meses após a implantação, época correspondente à estação chuvosa. Aspidosperma tomentosum e Stryphnodendron adstringens foram espécies que obtiveram os piores incrementos em diâmetro (Figura 3). 


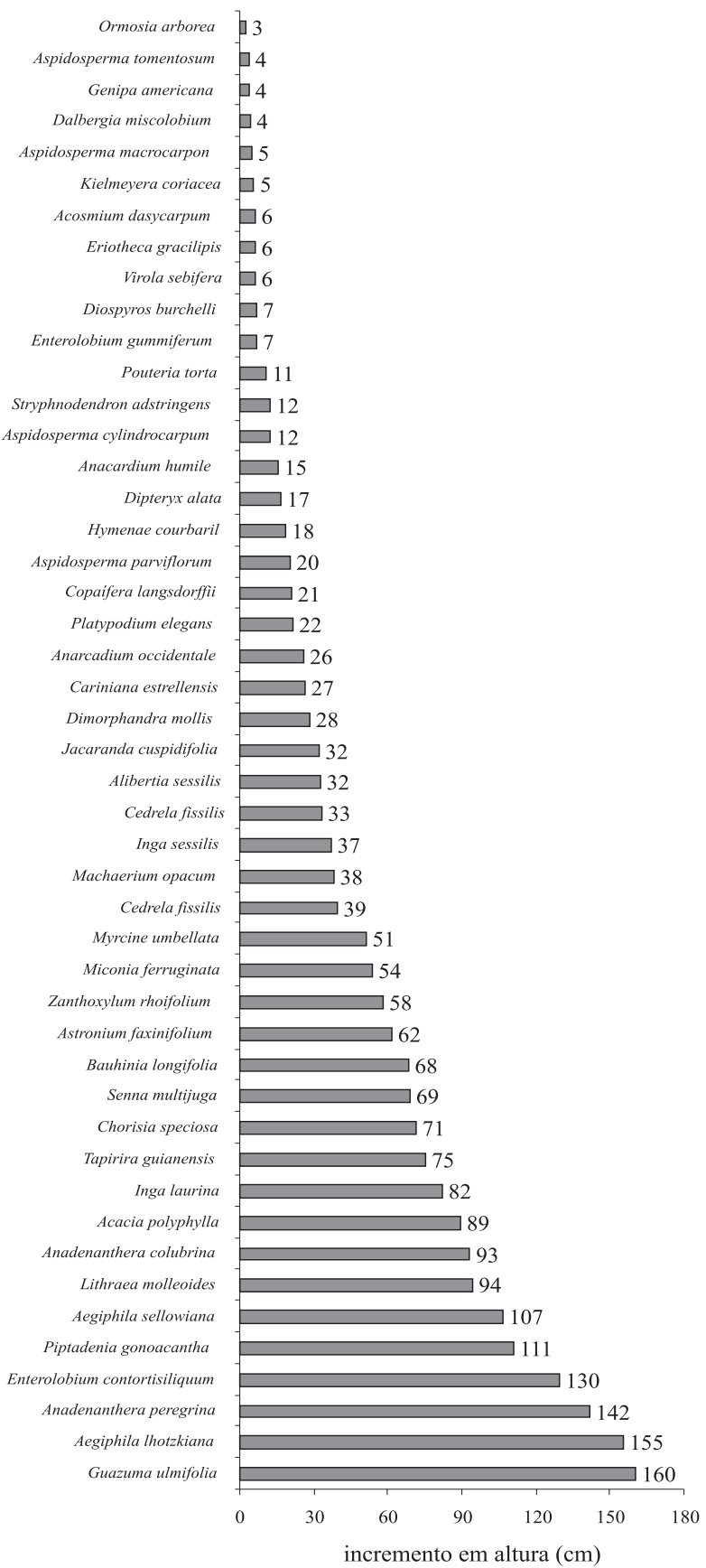

Figura 2 - Incremento em altura $(\mathrm{cm})$ das espécies após 13 meses da implantação na área no entorno do reservatório da Fazenda Mandaguari, no Município de Indianópolis, MG.

Figure 2 -Increase in height (cm) of the species 13 months after the implantation in the area around Mandaguari Farm's reservoir in the municipal district of Indianópolis, $M G$.

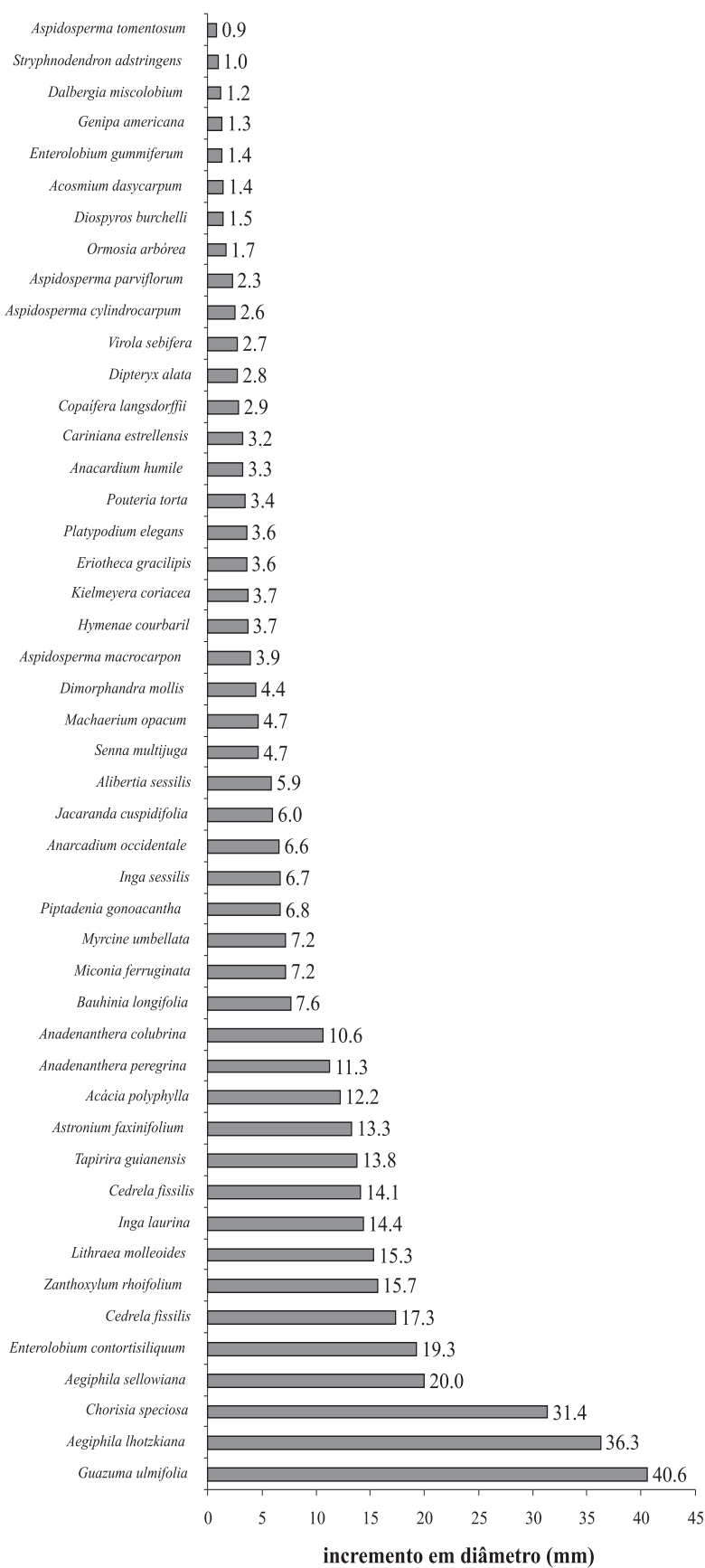

Figura 3 - Incremento em diâmetro $(\mathrm{mm})$ das espécies após 13 meses da implantação na área no entorno do reservatório da Fazenda Mandaguari, no Município da Indianópolis, MG.

Figure 3 - I Increase in the diameter $(\mathrm{mm})$ of the species 13 months after the implantation in the area around Mandaguari Farm's reservoir in the municipal district of Indianópolis, $M G$.

R. Árvore, Viçosa-MG, v.33, n.4, p.685-694, 2009 
As espécies foram distribuídas em três classes de incrementos em diâmetro; alto (diâmetros acima de $20 \mathrm{~mm}$ ), médio (entre 10,1 a 20,0 mm) e baixo (abaixo de 10,0 mm). A distribuição das espécies quanto ao incremento em diâmetro apresentou elevada concentração de espécies na classe de baixo incremento e redução acentuada no sentido das classes média e alta (Figura 3). A classe de baixo incremento em diâmetro compreendeu $68,1 \%$ das espécies, e as classes média e alta compreenderam 25,5 e 6,4\%, respectivamente.

De maneira geral, as espécies que apresentaram taxas de mortalidade entre 14,5 e 50\% tiveram de baixo a médio incremento em altura e diâmetro. Contudo, espécies como Guazuma ulmifolia e Aegiphila lhotzkiana, apesar de suas taxas de mortalidade serem relativamente altas, 50 e $28,6 \%$, respectivamente, agregaram características importantes de desenvolvimento que são a capacidade de cobertura (maiores alturas) e estabelecimento (maiores diâmetros) e, portanto, foram qualificadas como edificantes do processo de regeneração.

Chorisia speciosa, apesar do pouco destaque no incremento em altura, investiu mais no crescimento diamétrico, característica fundamental para o estabelecimento. Segundo Carneiro (1983), quanto menor for o valor da relação, maior será a capacidade das mudas em sobreviver e estabelecer-se. Sturion e Antunes (2000) acrescentaram que a relação altura da parte aérea e diâmetro do coleto constitui uma das características usadas para avaliar a qualidade de mudas florestais, pois, além de refletir o acúmulo de reservas, assegura maior resistência e melhor fixação do solo. Outras como Piptadenia gonoacantha e Anadenanthera peregrina investiram mais em altura. Diversos autores têm pesquisado o efeito de fatores como idade, densidade e posição sociológica sobre a dinâmica da distribuição diamétrica de diferentes espécies. É sabido que o crescimento acumulativo em diâmetro tende a aumentar com o avanço da idade, porém tende a reduzir com o incremento da densidade.

\section{CONCLUSÕES}

As espécies Anadenanthera colubrina, Aspidosperma tomentosum, Cedrela fissilis, Enterolobium contortisiliquum, Ormosia arborea e Zanthoxylum rhoifolium foram as que apresentaram maiores taxas de sobrevivência na área de estudo.
Guazuma ulmifolia e Aegiphila lhotzkiana, apesar de suas taxas de mortalidade relativamente altas, agregaram características importantes de desenvolvimento, como a capacidade de cobertura e estabelecimento, e, portanto, foram qualificadas como edificantes do processo de regeneração.

Chorisia speciosa, apesar do pouco destaque no incremento em altura, investiu mais no crescimento diamétrico, característica fundamental para o estabelecimento da espécie.

\section{REFERÊNCIAS}

AMO, S. R. Algunos aspectos de la influencia de la luz sobre el crecimiento de estados juveniles de especies primarias. In: GOMEZ-POMPA, A. \& $\mathrm{AMO}, \mathrm{S}$. R. Investigaciones sobre la regeneración de selvas altas en Veracruz, México. México, Alhambra Mexicana, 1985. v.2. p.79-92.

ANDRADE, L. A. B. L. Associação micorrízica e matéria orgânica no crescimento de Brachiara decumbens em estéril de mineração de ferro e bauxita. 1991. 48f. Dissertação (Mestrado emSolos e Nutrição de Plantas) - Universidade Federal de Viçosa, Viçosa, MG, 1991.

ARAÚJO, G. M.; GUIMARÃES, A. J. M.; NAKAJIMA, J. N. Fitossociologia de um remanescente de mata mesófila semidecídua urbana, Bosque John Kennedy, Araguari, MG, Brasil. Revista Brasileira de Botânica, v.20, n.1, p.67-77, 1997.

\section{BARBOSA, L. M. Modelos de repovoamento vegetal para proteção de sistemas hídricos em áreas degradadas dos diversos biomas no Estado de São Paulo. São Paulo: SMA/ FAPESP, 2002. 203p.}

BOARDMAN, N.K. Comparative photosynthesis of sun and shade plants. Annual Review of Plant Physiology, v.28, p.355-377, 1977.

BRASIL. Código Florestal Brasileiro lei no 4.771 , de 15 de setembro de 1965. Institui o novo código florestal. Diário Oficial da União, Brasília, DF, col. 2, p.9529, 16 set. de 1965. 
CARNEIRO, J. G. A. Variações na metodologia de produção de mudas florestais afetam os parâmetros morfológicos que afetam sua qualidade. Curitiba: FUPEP, 1983. 40p. (Série Técnica FUPEF, 12).

EMPRESA BRASILEIRA DE PESQUISA AGROPECUÁRIA - EMBRAPA. EMPRESA DE PESQUISAAGROPECUÁRIADE MINAS GERAIS - EPAMIG. Levantamento de reconhecimento de média intensidade de solos e avaliação da aptidão agrícola das terras no Triângulo Mineiro. Rio de Janeiro: Embrapa/SNLCS, 1982. 526p. (Boletim de Pesquisa, 1).

FELFILI, J. M. et al. Recuperação de matas de galeria. Planaltina: Embrapa Cerrados, 2000. 45p. (Documentos, 21).

FERREIRA, D. A. C.; DIAS, H. C. T. Situação da mata ciliar do ribeirão são Bartolomeu em Viçosa, MG. Revista Árvore, v.28, n.4, p.617-623, 2004.

FONSECA, C. E. L. et al. Recuperação da vegetação de Matas de Galeria: estudos de caso no Distrito Federal e entorno. In: RIBEIRO, J. F.; FONSECA, C. E. L.; SOUZA-SILVA, J. C. (Eds.). Cerrado - caracterização e recuperação de matas de galeria. Planaltina: [s.n.], 2001. v.1. p.815-867.

GISLER, C. V. T.; BARBOSA, L. M. Estrutura e função de mata ciliar implantada em Santa Cruz das Palmeiras, SP. In: SIMPÓSIO NACIONAL DE RECUPERAÇÃO DE AREAS DEGRADADAS, 4., 2000, Blumenau. Anais... Blumenau: Sociedade Brasileira de Recuperação de áreas Degradadas, 2000. CD ROM.

HANBA, Y. T.; KOGAMI, H.; TESASHIMA, L. The effects of growth irradiance on leaf anatomy and photosynthesis in Acer species differing in light demand. Plant Cell and

Environment, v.25, n.8, p.1021-1030, 2002.

HOLL, K. D. et al. Tropical montane forest restoration in Costa Rica: Overcoming barriers to dispersal and establishment. Restoration Ecology, v.8, n.4, p.339-349, 2000.
KAGEYAMA, P. Y.; GANDARA, F. B. Recuperação de áreas ciliares. In: RODRIGUES, R. R.; LEITÃO FILHO, H. F. Matas ciliares: conservação e recuperação. São Paulo: Universidade de São Paulo/ Fapesp, 2000. p.249-269.

KAGEYAMA, P. Y. Conservação "in situ” de recursos genéticos de plantas. IPEF, v.35, p.7-37, 1987.

KÖPPEN, W. Climatologia. México: Fundo de Cultura Económica, 1948. p.553-557.

MACEDO, A. C.; KAGEYAMA, P. Y.; COSTA, L. G. S. Revegetação: matas ciliares e de proteção ambiental. Revisado e ampliado por KAGEYAMA, P. Y.; DA COSTA, L. G. S. São Paulo: Governo do estado de São Paulo, Secretária do Meio Ambiente/Fundação Florestal, 1993. 27p.

MARTINS, S. V. Recuperação de matas de ciliares. Viçosa,MG: Aprenda Fácil, 2001. 143p.

MARTINS, C. R.; LEITE, L. L.; HARIDASAN, M. Recuperação de uma área degradada pela mineração de cascalho com o uso de gramíneas nativas. Revista Árvore, v.25, n.2, p.157-166, 2001.

MENEGHELLO, G. E.; MATTEI, V. L. Semeadura direta de timbaúba (Enterolobium controtisiliquum), canafístula (Peltophorum dubium) e cedro (Cedrela fissilis) em campos abandonados. Ciências Florestais, v.14, n.2, p.21-27, 2004.

OLIVEIRA FILHO, A. T. et al. Estrutura fitossociológica e variáveis ambientais em um trecho de mata ciliar do córrego dos Vilas Boas, reserva biológica do Poço Bonito, Lavras (MG). Revista Brasileira de Botânica, v.17, n.1, p.67-85, 1994.

OLIVEIRA FILHO, A. T.; MARTINS, F. R. Distribuição, caracterização e composição florística das formações vegetais da região da Salgadeira, na Chapada do Guimarães (MT). Revista Brasileira de Botânica, v.9, n.2, p.207-223, 1986.

R. Árvore, Viçosa-MG, v.33, n.4, p.685-694, 2009 
OLIVEIRA FILHO, A. T. Composição florística e estrutura comunitária da floresta de galeria do córrego da Paciência, Cuiabá (MT). Acta

Botanica Brasilica, v.3, n.1, p.91-112, 1989.

PEDRALLI, G.; TEIXEIRA, M. C. B. Levantamento florístico e principais fisionomias na Estação de Pesquisa e Desenvolvimento Ambiental de Peti, Santa Bárbara, MG, Brasil. Iheringia, Série Botânica, v.48, n.1, p.15-40, 1997.

PINTO-COELHO, R. M. Fundamentos em ecologia. Porto Alegre: Artes Médicas do Sul. 2000. p.87-88.

REZENDE, A. V. Importância das Matas de Galeria: manutenção e recuperação. In: RIBEIRO, J. F. (Ed.). Cerrado: Matas de Galeria. Planaltina: Embrapa, 1998. p.3-16.

RIBEIRO, J. F.; WALTER, B. M. T. Fitofisionomias do Bioma Cerrado. In: SANO, S. M.; ALMEIDA, S. P. Cerrado: ambiente e flora. Planaltina: Embrapa-CPAC, 1998. p.89-166.
SCHLÜTER, U. et al. Photosynthetic performance of an Arabidopis mulant with elevated stomatal density (sdd 1-1) under different light regimes. Journal of Experimental Botany, v.54, n.383, p.867-874, 2003.

STURION, J. A.; ANTUNES, B. M. A. Produção de mudas de espécies florestais. In: GALVÃO, A. P. M. Reflorestamento de propriedades rurais para fins produtivos e ambientais. Brasília: Embrapa, 2000. p.125-150.

Whatley, J. M.; Whatley, F. R. A luz e a vida das plantas. São Paulo, EPU/EDUSP, 1982. 101p. (Temas de Biologia, 30).

WHITE, P. S.; PICKETT, S. T. A. Natural disturbance and patch dynamics: an introduction. In: WHITE, P. S.; PICKETT, S. T. A. (Eds.).The ecology of natural disturbance and patch dynamics. New York: Academic Press, 1985. p.3-13. 